\title{
Sharp trapezoid and mid-point type inequalities on closed balls in $\mathbb{R}^{3}$
}

M. Rostamian Delavar ${ }^{1 *}$

\section{"Correspondence:}

m.rostamian@ub.ac.ir

'Department of Mathematics,

Faculty of Basic Sciences, University

of Bojnord, Bojnord, Iran

\section{Abstract}

This paper deals with some trapezoid and mid-point type inequalities on closed balls in $\mathbb{R}^{3}$. Three kinds of functions are considered: convex, Lipschitz, and bounded functions. The spherical coordinates are used to obtain sharp inequalities. Also a reverse result is given for the right-hand side of Hermite-Hadamard's inequality obtained on closed balls in $\mathbb{R}^{3}$.

MSC: Primary 26B25; 26B15; 26D15; secondary 26A51

Keywords: Hermite-Hadamard inequality; Trapezoid type inequality; Mid-point type inequality; Spherical coordinates

\section{Introduction and preliminaries}

Consider the closed ball $\overline{\mathcal{B}}(\mathcal{C}, R)$ in the space $\mathbb{R}^{3}$ with center $\mathcal{C}=(a, b, c) \in \mathbb{R}^{3}$ and radius $R>0$ defined as

$$
\overline{\mathcal{B}}(\mathcal{C}, R)=\left\{(x, y, z) \in \mathbb{R}^{3} \mid(x-a)^{2}+(y-b)^{2}+(z-c)^{2} \leq R^{2}\right\} .
$$

Also consider $\sigma(\mathcal{C}, R)$ as the boundary (the surface) of $\bar{B}(\mathcal{C}, R)$, i.e.,

$$
\sigma(\mathcal{C}, R)=\left\{(x, y, z) \in \mathbb{R}^{3} \mid(x-a)^{2}+(y-b)^{2}+(z-c)^{2}=R^{2}\right\} .
$$

The following result has been proved in [1], which is the Hermite-Hadamard's inequality for convex functions defined on closed ball $\overline{\mathcal{B}}(\mathcal{C}, R)$.

Theorem 1.1 Let $f: \overline{\mathcal{B}}(\mathcal{C}, R) \rightarrow \mathbb{R}$ be a convex mapping on the ball $\overline{\mathcal{B}}(\mathcal{C}, R)$. Then we have the inequality

$$
f(\mathcal{C}) \leq \frac{1}{v(\overline{\mathcal{B}}(\mathcal{C}, R))} \iiint_{\overline{\mathcal{B}}(\mathcal{C}, R)} f(x, y, z) d x d y d z \leq \frac{1}{\sigma(\bar{B}(\mathcal{C}, R))} \iint_{\sigma(\mathcal{C}, R)} f(x, y, z) d \sigma,
$$

where $v(\overline{\mathcal{B}}(\mathcal{C}, R))=\frac{4 \pi R^{3}}{3}$ and $\sigma(\bar{B}(\mathcal{C}, R))=\frac{1}{4 \pi R^{2}}$.

(c) The Author(s) 2020. This article is licensed under a Creative Commons Attribution 4.0 International License, which permits use, sharing, adaptation, distribution and reproduction in any medium or format, as long as you give appropriate credit to the original author(s) and the source, provide a link to the Creative Commons licence, and indicate if changes were made. The images or other third party material in this article are included in the article's Creative Commons licence, unless indicated otherwise in a credit line to the material. If material is not included in the article's Creative Commons licence and your intended use is not permitted by statutory regulation or exceeds the permitted use, you will need to obtain permission directly from the copyright holder. To view a copy of this licence, visit http://creativecommons.org/licenses/by/4.0/. 
The main purpose of this paper is estimating two bounds $\mathcal{B}_{1}$ and $\mathcal{B}_{2}$ such that

$$
\left|\frac{1}{\frac{4}{3} \pi R^{3}} \iiint_{\bar{B}(\mathcal{C}, R)} f(x, y, z) d V-f(\mathcal{C})\right| \leq \mathcal{B}_{1}
$$

and

$$
\left|\frac{1}{4 \pi R^{2}} \iint_{\sigma(\mathcal{C}, R)} f(x, y, z) d \sigma-\frac{1}{\frac{4}{3} \pi R^{3}} \iiint_{\bar{B}(\mathcal{C}, R)} f(x, y, z) d V\right| \leq \mathcal{B}_{2}
$$

Depending on the properties of the function $f$ and the radius $R$, different values will be obtained for $\mathcal{B}_{1}$ and $\mathcal{B}_{2}$.

We call (2) a mid-point type inequality due to the following result obtained in [2] and interpretation of Fig. 1.

Theorem 1.2 Let $f: I^{\circ} \subseteq \mathbb{R} \rightarrow \mathbb{R}$ be a differentiable mapping on $I^{\circ}, a, b \in I^{\circ}$ with $a<b$. If $f^{\prime} \mid$ is convex on $[a, b]$, then we have

$$
\left|\int_{a}^{b} f(x) d x-(b-a) f\left(\frac{a+b}{2}\right)\right| \leq \frac{1}{8}(b-a)^{2}\left(\left|f^{\prime}(a)\right|+\left|f^{\prime}(b)\right|\right) .
$$

According to (4), we have an estimate for the difference between the area under the graph of $f$, i.e., $\int_{a}^{b} f(x) d x$, and the area of rectangle $a b c d$, i.e., $(b-a) f\left(\frac{a+b}{2}\right)$ (see Fig. 1).

Also we call (3) a trapezoid type inequality due to the following result and Fig. 2.

Figure 1 Mid-point type inequality

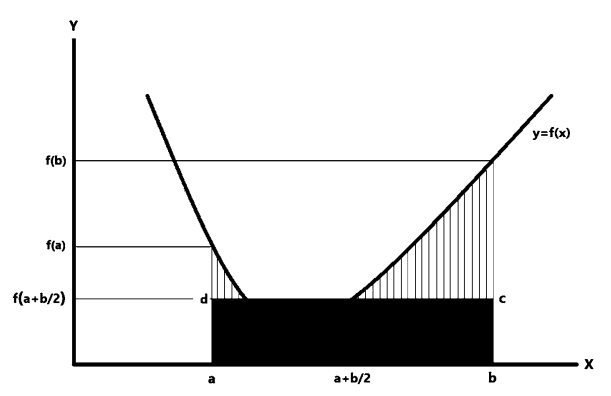

Figure 2 Trapezoid type inequality

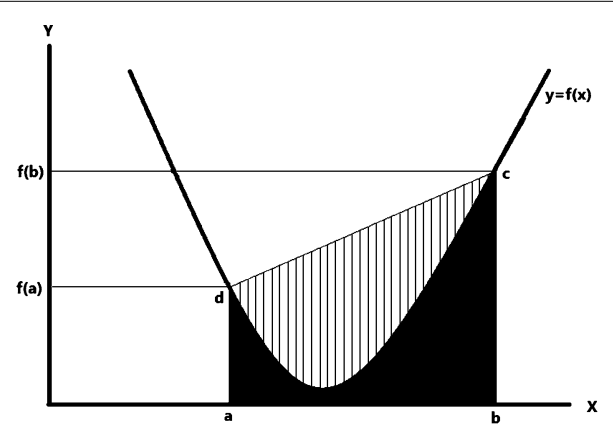


Theorem 1.3 ([3]) Let $f: I^{\circ} \subseteq \mathbb{R} \rightarrow \mathbb{R}$ be a differentiable mapping on $I^{\circ}, a, b \in I^{\circ}$ with $a<b$. If $\left|f^{\prime}\right|$ is convex on $[a, b]$, then the following inequality holds:

$$
\left|\int_{a}^{b} f(x) d x-(b-a) \frac{f(a)+f(b)}{2}\right| \leq \frac{1}{8}(b-a)^{2}\left(\left|f^{\prime}(a)\right|+\left|f^{\prime}(b)\right|\right) .
$$

According to (5), we can estimate the difference between the area of trapezoid $a b c d$, i.e., $(b-a) \frac{f(a)+f(b)}{2}$, and the area under the graph of $f$ (see Fig. 2).

Note that to obtain (4) and (5), the absolute values of the derivative of $f$ at boundary points of interval $[a, b]$ play a fundamental role. For more results about HermiteHadamard's inequality, we refer an interested reader to [4-18] and the references therein.

Before presenting our main results, here we obtain a new representation of (1) and also give a reverse type theorem.

If we consider a convex function $f: \overline{\mathcal{B}}(\mathcal{C}, R) \rightarrow \mathbb{R}$ and the change of coordinates

$$
\begin{aligned}
& \mathcal{T}: \bar{D}((a, b), R) \times[0,1] \rightarrow \overline{\mathcal{B}}(\mathcal{C}, R), \\
& \mathcal{T}(x, y, \lambda)=\left(x, y,(2 \lambda-1) \sqrt{R^{2}-x^{2}-y^{2}}\right),
\end{aligned}
$$

where $\bar{D}((a, b), R)$ is a closed disk centered at the point $(a, b)$ having radius $R>0$, then we obtain

$$
\begin{aligned}
& \iiint_{\overline{\mathcal{B}}(\mathcal{C}, R)} f(x, y, z) d V \\
& =2 \int_{-R}^{R} \int_{-\sqrt{R^{2}-x^{2}}}^{\sqrt{R^{2}-x^{2}}} \int_{0}^{1} f\left((1-\lambda)\left(x, y,-\sqrt{R^{2}-x^{2}-y^{2}}\right)+\lambda\left(x, y, \sqrt{R^{2}-x^{2}-y^{2}}\right)\right) \\
& \quad \times \sqrt{R^{2}-x^{2}-y^{2}} d \lambda d y d x \\
& \leq 2 \int_{-R}^{R} \int_{-\sqrt{R^{2}-x^{2}}}^{\sqrt{R^{2}-x^{2}}} \int_{0}^{1}(1-\lambda) f\left(x, y,-\sqrt{R^{2}-x^{2}-y^{2}}\right) \sqrt{R^{2}-x^{2}-y^{2}} d \lambda d y d x \\
& \quad+2 \int_{-R}^{R} \int_{-\sqrt{R^{2}-x^{2}}}^{\sqrt{R^{2}-x^{2}}} \int_{0}^{1} \lambda f\left(x, y, \sqrt{R^{2}-x^{2}-y^{2}}\right) \sqrt{R^{2}-x^{2}-y^{2}} d \lambda d y d x \\
& =\int_{-R}^{R} \int_{-\sqrt{R^{2}-x^{2}}}^{\sqrt{R^{2}-x^{2}}} f\left(x, y,-\sqrt{R^{2}-x^{2}-y^{2}}\right) \sqrt{R^{2}-x^{2}-y^{2}} d y d x \\
& \quad+\int_{-R}^{R} \int_{-\sqrt{R^{2}-x^{2}}}^{\sqrt{R^{2}-x^{2}}} f\left(x, y, \sqrt{R^{2}-x^{2}-y^{2}}\right) \sqrt{R^{2}-x^{2}-y^{2}} d y d x .
\end{aligned}
$$

Choosing $z=\sqrt{R^{2}-x^{2}-y^{2}}$ in the latter integrals, the fact that $\sqrt{1+\left(\frac{\partial z}{\partial x}\right)^{2}+\left(\frac{\partial z}{\partial y}\right)^{2}}=$ $\frac{R}{\sqrt{R^{2}-x^{2}-y^{2}}}=\frac{R}{z}$, and using the surface integral formula for $\sigma(\mathcal{C}, R)$ imply that

$$
\iiint_{\overline{\mathcal{B}}(\mathcal{C}, R)} f(x, y, z) d V \leq \frac{1}{R} \iint_{\sigma(\mathcal{C}, R)} f(x, y, z) z^{2} d \sigma .
$$

Inequality (8) gives another representation for (1).

In a special case for a convex function $f: \bar{D}((a, b), R) \rightarrow \mathbb{R}$ we get

$$
\iint_{\bar{D}((a, b), R)} f(x, y) d A \leq \frac{1}{R} \int_{\sigma((a, b), R)} f(x, y) y^{2} d \sigma .
$$


Now for a reverse type result, consider a continuous function $f$ defined on a convex subset $\mathcal{V} \subset \mathbb{R}^{3}$ such that (8) holds for all closed balls included in $\mathcal{V}$. Then $f$ is convex on $\mathcal{V}$ because otherwise there would exist $\mathcal{X}, \mathcal{Y} \in \mathcal{V}$ and $\lambda \in(0,1)$ such that

$$
f(\lambda \mathcal{X}+(1-\lambda) \mathcal{Y})>\lambda f(\mathcal{X})+(1-\lambda) f(\mathcal{Y})
$$

Since $f$ is continuous on $\mathcal{V}$, we can find $R>0$ and a point $\mathcal{Z}=(\bar{a}, \bar{b}, \bar{c})$ in a convex combination of $\mathcal{X}$ and $\mathcal{Y}$ such that (9) holds on the whole of $\overline{\mathcal{B}}((\bar{a}, \bar{b}, \bar{c}), R) \subset \mathcal{V}$. So by the change of coordinates (6) and structure presented in (7) for $\bar{D}((\bar{a}, \bar{b}), R)$ and $\overline{\mathcal{B}}((\bar{a}, \bar{b}, \bar{c}), R)$, we obtain that

$$
\iiint_{\overline{\mathcal{B}}((\bar{a}, \bar{b}, \bar{c}, R)} f(x, y, z) d V>\frac{1}{R} \iint_{\sigma((\bar{a}, \bar{b}, \bar{c}), R)} f(x, y, z) z^{2} d \sigma
$$

which is a contradiction and this proves the convexity of $f$ on $\mathcal{V}$.

In the following sections we consider convex, Lipschitz, and bounded functions to obtain some trapezoid and mid-point type inequalities on a closed ball. We use the spherical coordinates in calculating the integrals.

\section{Convex functions}

In this section we obtain trapezoid and mid-point type inequalities for the case that the partial derivative absolute values of a considered function with respect to the radius in spherical coordinates is convex. We need the following lemma.

Lemma 2.1 For an integrable function $f: \overline{\mathcal{B}}(\mathcal{C}, R) \rightarrow \mathbb{R}$, we have

$$
\begin{aligned}
& \iiint_{\overline{\mathcal{B}}(\mathcal{C}, R)} f(x, y, z) d V \\
& =\int_{0}^{2 \pi} \int_{0}^{\pi} \int_{0}^{R} f(a+\rho \cos \theta \sin \varphi, b+\rho \sin \theta \sin \varphi, c+\rho \cos \varphi) \\
& \quad \times \rho^{2} \sin \varphi d \rho d \varphi d \theta,
\end{aligned}
$$

and

$$
\begin{aligned}
& \iint_{\sigma(\mathcal{C}, R)} f(x, y, z) d \sigma \\
& =\int_{0}^{2 \pi} \int_{0}^{\pi} f(a+R \cos \theta \sin \varphi, b+R \sin \theta \sin \varphi, c+R \cos \varphi) R^{2} \sin \varphi d \varphi d \theta \\
& =\frac{3}{R} \int_{0}^{2 \pi} \int_{0}^{\pi} \int_{0}^{R} f(a+R \cos \theta \sin \varphi, b+R \sin \theta \sin \varphi, c+R \cos \varphi) \\
& \quad \times \rho^{2} \sin \varphi d \rho d \varphi d \theta .
\end{aligned}
$$

Proof Consider the spherical transformation

$$
\left\{\begin{array}{l}
x(\rho, \varphi, \theta)=a+\rho \cos \theta \sin \varphi, \\
y(\rho, \varphi, \theta)=b+\rho \sin \theta \sin \varphi, \quad \rho \in[0, R], \varphi \in[0, \pi], \theta \in[0,2 \pi] . \\
z(\rho, \varphi, \theta)=c+\rho \cos \varphi,
\end{array}\right.
$$


It is obvious that the Jacobian of this transformation is $J=\rho^{2} \sin \varphi$. So we have (10).

For (11), consider the curve $\eta:[0, \pi] \times[0,2 \pi] \rightarrow \mathbb{R}^{3}$ defined by

$$
\eta(\theta, \varphi):\left\{\begin{array}{l}
x(\varphi, \theta)=a+R \cos \theta \sin \varphi, \\
y(\varphi, \theta)=b+R \sin \theta \sin \varphi, \\
z(\varphi, \theta)=c+R \cos \varphi,
\end{array} \quad \varphi \in[0, \pi], \theta \in[0,2 \pi] .\right.
$$

It is clear that $\eta([0, \pi] \times[0,2 \pi])=\sigma(C, R)$ and then by integrating with respect to the surface (arc length) we get

$$
\begin{aligned}
& \iint_{\sigma(\mathcal{C}, R)} f(x, y, z) d \sigma \\
& =\iint_{\sigma(\mathcal{C}, R)} f(\eta) d l(\eta) \\
& =\int_{0}^{2 \pi} \int_{0}^{\pi} f(x(\varphi, \theta), y(\varphi, \theta), z(\varphi, \theta)) \\
& \quad \times\left(\left[\frac{\partial x(\varphi, \theta)}{\partial \theta}\right]^{2}+\left[\frac{\partial y(\varphi, \theta)}{\partial \theta}\right]^{2}+\left[\frac{\partial z(\varphi, \theta)}{\partial \theta}\right]^{2}\right)^{\frac{1}{2}} \\
& \quad \times\left(\left[\frac{\partial x(\varphi, \theta)}{\partial \varphi}\right]^{2}+\left[\frac{\partial y(\varphi, \theta)}{\partial \varphi}\right]^{2}+\left[\frac{\partial z(\varphi, \theta)}{\partial \varphi}\right]^{2}\right)^{\frac{1}{2}} d \varphi d \theta \\
& =\int_{0}^{2 \pi} \int_{0}^{\pi} f(a+R \cos \theta \sin \varphi, b+R \sin \theta \sin \varphi, c+R \cos \varphi) R^{2} \sin \varphi d \varphi d \theta \\
& =\frac{3}{R} \int_{0}^{2 \pi} \int_{0}^{\pi} \int_{0}^{R} f(a+R \cos \theta \sin \varphi, b+R \sin \theta \sin \varphi, c+R \cos \varphi) \rho^{2} \sin \varphi d \rho d \varphi d \theta .
\end{aligned}
$$

This proves (11).

The following is a sharp trapezoid type inequality related to (1), where we consider a function with convex partial derivative (with respect to the radius $\rho$ ) absolute values defined on $\overline{\mathcal{B}}(\mathcal{C}, R)$.

Theorem 2.2 For $\mathcal{V} \subset \mathbb{R}^{3}$, suppose that $\overline{\mathcal{B}}(\mathcal{C}, R) \subset \mathcal{V}^{\circ}$ where $\mathcal{V}^{\circ}$ is the interior of $\mathcal{V}$. Consider $f: \overline{\mathcal{B}}(\mathcal{C}, R) \rightarrow \mathbb{R}$ which has continuous partial derivatives with respect to the variables $\rho, \varphi$, and $\theta$ on $\overline{\mathcal{B}}(\mathcal{C}, R)$ in spherical coordinates. If $\left|\frac{\partial f}{\partial \rho}\right|$ is convex on $\overline{\mathcal{B}}(\mathcal{C}, R)$, then

$$
\begin{aligned}
& \left|\frac{1}{4 \pi R^{2}} \iint_{\sigma(\mathcal{C}, R)} f(x, y, z) d \sigma-\frac{1}{\frac{4}{3} \pi R^{3}} \iiint_{\overline{\mathcal{B}}(\mathcal{C}, R)} f(x, y, z) d V\right| \\
& \quad \leq \frac{1}{16 \pi R} \iint_{\sigma(\mathcal{C}, R)}\left|\frac{\partial f}{\partial \rho}\right|(x, y, z) d \sigma .
\end{aligned}
$$

Furthermore, inequality (12) is sharp.

Proof For fixed $\varphi \in[0, \pi]$ and $\theta \in[0,2 \pi]$ and arbitrary $\rho \in[0, R]$, since

$$
\left(\left[\frac{\partial x(\rho, \varphi, \theta)}{\partial \rho}\right]^{2}+\left[\frac{\partial y(\rho, \varphi, \theta)}{\partial \rho}\right]^{2}+\left[\frac{\partial z(\rho, \varphi, \theta)}{\partial \rho}\right]^{2}\right)^{\frac{1}{2}}=1,
$$


by integration by parts we have

$$
\begin{aligned}
\int_{0}^{R} & \frac{\partial f}{\partial \rho}(a+\rho \cos \theta \sin \varphi, b+\rho \sin \theta \sin \varphi, c+\rho \cos \varphi) \rho^{3} \sin \varphi d \rho \\
= & R^{3} f(a+R \cos \theta \sin \varphi, b+R \sin \theta \sin \varphi, c+R \cos \varphi) \\
& -3 \int_{0}^{R} f(a+\rho \cos \theta \sin \varphi, b+\rho \sin \theta \sin \varphi, c+\rho \cos \varphi) \rho^{2} \sin \varphi d \rho .
\end{aligned}
$$

So integrating with respect to $\varphi \in[0, \pi]$ and $\theta \in[0,2 \pi]$ in (13), along with (10) and (11) obtained in Lemma 2.1 and the convexity of $\left|\frac{\partial f}{\partial \rho}\right|$ on $\overline{\mathcal{B}}(\mathcal{C}, R)$, implies that

$$
\begin{aligned}
&\left|R \iint_{\sigma(\mathcal{C}, R)} f(x, y, z) d \sigma-3 \iiint_{\overline{\mathcal{B}}(\mathcal{C}, R)} f(x, y, z) d V\right| \\
& \leq \int_{0}^{2 \pi} \int_{0}^{\pi} \int_{0}^{R}\left|\frac{\partial f}{\partial \rho}\right|(a+\rho \cos \theta \sin \varphi, b+\rho \sin \theta \sin \varphi, c+\rho \cos \varphi) \rho^{3} \sin \varphi d \rho d \varphi d \theta \\
& \leq \int_{0}^{2 \pi} \int_{0}^{\pi} \int_{0}^{R}\left|\frac{\partial f}{\partial \rho}\right|\left(\left(1-\frac{\rho}{R}\right)(a, b, c)\right. \\
&\left.+\frac{\rho}{R}(a+R \cos \theta \sin \varphi, b+R \sin \theta \sin \varphi, c+R \cos \varphi)\right) \\
& \times \rho^{3} \sin \varphi d \rho d \varphi d \theta \\
& \leq \int_{0}^{2 \pi} \int_{0}^{\pi} \int_{0}^{R} \rho^{3}\left(1-\frac{\rho}{R}\right)\left|\frac{\partial f}{\partial \rho}\right|(a, b, c) \sin \varphi d \rho d \varphi d \theta \\
&+\int_{0}^{2 \pi} \int_{0}^{\pi} \int_{0}^{R} \frac{\rho^{4}}{R}\left|\frac{\partial f}{\partial \rho}\right|(a+R \cos \theta \sin \varphi, b+R \sin \theta \sin \varphi, c+R \cos \varphi) \\
& \times \sin \varphi d \rho d \varphi d \theta \\
&= \frac{\pi R^{4}}{5}\left|\frac{\partial f}{\partial \rho}\right|(a, b, c)+\frac{R^{2}}{5} \iint_{\sigma(\mathcal{C}, R)}\left|\frac{\partial f}{\partial \rho}\right|(x, y, z) d \sigma
\end{aligned}
$$

By considering the left-hand side of (1) for $\left|\frac{\partial f}{\partial \rho}\right|$ and applying it in (14), we have

$$
\begin{aligned}
\mid R & \iint_{\sigma(\mathcal{C}, R)} f(x, y, z) d \sigma-3 \iiint_{\overline{\mathcal{B}}(\mathcal{C}, R)} f(x, y, z) d V \mid \\
& \leq \frac{R^{2}}{20} \iint_{\sigma(\mathcal{C}, R)}\left|\frac{\partial f}{\partial \rho}\right|(x, y, z) d \sigma+\frac{R^{2}}{5} \iint_{\sigma(\mathcal{C}, R)}\left|\frac{\partial f}{\partial \rho}\right|(x, y, z) d \sigma \\
& =\frac{R^{2}}{4} \iint_{\sigma(\mathcal{C}, R)}\left|\frac{\partial f}{\partial \rho}\right|(x, y, z) d \sigma .
\end{aligned}
$$

By dividing (15) with $4 \pi R^{3}$, we obtain the desired result (12).

To show the sharpness of (12), consider the function $f: \overline{\mathcal{B}}(\mathcal{C}, R) \rightarrow \mathbb{R}$ defined as

$$
f(x, y, z)=R-\sqrt{(x-a)^{2}+(y-b)^{2}+(z-c)^{2}} .
$$


Using spherical coordinates, we have $f(\rho, \varphi, \theta)=R-\rho$, for $\rho \in[0, R], \varphi \in[0, \pi]$ and $\theta \in$ $[0,2 \pi]$. With some calculations we obtain that

$$
\begin{aligned}
& \frac{1}{\frac{4}{3} \pi R^{3}} \iiint_{\overline{\mathcal{B}}(\mathcal{C}, R)} f(x, y, z) d V \\
& \quad=\frac{1}{\frac{4}{3} \pi R^{3}} \int_{0}^{2 \pi} \int_{0}^{\pi} \int_{0}^{R}(R-\rho) \rho^{2} \sin \varphi d \rho d \varphi d \theta=\frac{R}{4},
\end{aligned}
$$

and

$$
\iint_{\sigma(\mathcal{C}, R)} f(x, y, z) d \sigma=0
$$

On the other hand, since $\left|\frac{\partial f}{\partial \rho}\right|=1$,

$$
\frac{1}{16 \pi R} \iint_{\sigma(\mathcal{C}, R)}\left|\frac{\partial f}{\partial \rho}\right|(x, y, z) d \sigma=\frac{R}{4}
$$

From (16) and (17) we have the sharpness of (12).

Now we obtain the midpoint type inequality related to (1), where the partial derivative absolute value of considered function defined on $\overline{\mathcal{B}}(\mathcal{C}, R)$ is convex.

Theorem 2.3 Suppose that $\overline{\mathcal{B}}(\mathcal{C}, R) \subset \mathcal{V}^{\circ}$, where $\mathcal{V} \subset \mathbb{R}^{3}$. Consider $f: \overline{\mathcal{B}}(\mathcal{C}, R) \rightarrow \mathbb{R}$ which has continuous partial derivatives with respect to the variables $\rho$, $\varphi$, and $\theta$ on $\overline{\mathcal{B}}(\mathcal{C}, R)$ in spherical coordinates. If $\left|\frac{\partial f}{\partial \rho}\right|$ is convex on $\overline{\mathcal{B}}(\mathcal{C}, R)$, then

$$
\left|\frac{1}{\frac{4}{3} \pi R^{3}} \iiint_{\overline{\mathcal{B}}(\mathcal{C}, R)} f(x, y, z) d V-f(\mathcal{C})\right| \leq \frac{5}{16 \pi R} \iint_{\sigma(\mathcal{C}, R)}\left|\frac{\partial f}{\partial \rho}\right|(x, y, z) d \sigma .
$$

Proof Similar to the proof of Theorem 2.2, for fixed $\varphi \in[0, \pi]$ and $\theta \in[0,2 \pi]$, we have

$$
\begin{aligned}
& \int_{0}^{R} \frac{\partial f}{\partial \rho}(a+\rho \cos \theta \sin \varphi, b+\rho \sin \theta \cos \varphi, c+\rho \cos \varphi) \sin \varphi d \rho \\
& \quad=f(a+R \cos \theta \sin \varphi, b+R \sin \theta \sin \varphi, c+R \cos \varphi) \sin \varphi-f(\mathcal{C}) \sin \varphi
\end{aligned}
$$

Integration with respect to the variables $\varphi \in[0, \pi]$ and $\theta \in[0,2 \pi]$ in (19) implies that

$$
\begin{aligned}
& \int_{0}^{2 \pi} \int_{0}^{\pi} \int_{0}^{R} \frac{\partial f}{\partial \rho}(a+\rho \cos \theta \sin \varphi, b+\rho \sin \theta \cos \varphi, c+\rho \cos \varphi) \sin \varphi d \rho d \varphi d \theta \\
& =\frac{1}{R^{2}} \iint_{\sigma(\mathcal{C}, R)} f(x, y, z) d \sigma-4 \pi f(\mathcal{C}) .
\end{aligned}
$$

So from the convexity of $\left|\frac{\partial f}{\partial \rho}\right|$ we get

$$
\left|\frac{1}{4 \pi R^{2}} \iint_{\sigma(\mathcal{C}, R)} f(x, y, z) d \sigma-f(\mathcal{C})\right|
$$




$$
\begin{aligned}
\leq & \frac{1}{4 \pi} \int_{0}^{2 \pi} \int_{0}^{\pi} \int_{0}^{R}\left|\frac{\partial f}{\partial \rho}\right|(a+\rho \cos \theta \sin \varphi, b+\rho \sin \theta \sin \varphi, c+\rho \cos \varphi) \sin \varphi d \rho d \varphi d \theta \\
\leq & \frac{1}{4 \pi} \int_{0}^{2 \pi} \int_{0}^{\pi} \int_{0}^{R}\left(1-\frac{\rho}{R}\right)\left|\frac{\partial f}{\partial \rho}\right|(\mathcal{C}) \sin \varphi d \rho d \varphi d \theta \\
& +\frac{1}{4 \pi} \int_{0}^{2 \pi} \int_{0}^{\pi} \int_{0}^{R} \frac{\rho}{R}\left|\frac{\partial f}{\partial \rho}\right|(a+R \cos \theta \sin \varphi, b+R \sin \theta \sin \varphi, c+R \cos \varphi) \\
& \times \sin \varphi d \rho d \varphi d \theta \\
= & \frac{R}{2}\left|\frac{\partial f}{\partial \rho}\right|(\mathcal{C})+\frac{1}{8 \pi R} \iint_{\sigma(\mathcal{C}, R)}\left|\frac{\partial f}{\partial \rho}\right|(x, y, z) d \sigma .
\end{aligned}
$$

It follows from triangle inequality, (20), (12) and (1)(for $\left.\left|\frac{\partial f}{\partial \rho}\right|\right)$ that

$$
\begin{aligned}
& \left|\frac{1}{\frac{4}{3} \pi R^{3}} \iiint_{\overline{\mathcal{B}}(\mathcal{C}, R)} f(x, y, z) d V-f(\mathcal{C})\right| \\
& \quad \leq \frac{1}{16 \pi R} \iint_{\sigma(\mathcal{C}, R)}\left|\frac{\partial f}{\partial \rho}\right|(x, y, z) d \sigma+\frac{R}{2}\left|\frac{\partial f}{\partial \rho}\right|(\mathcal{C})+\frac{1}{8 \pi R} \iint_{\sigma(\mathcal{C}, R)}\left|\frac{\partial f}{\partial \rho}\right|(x, y, z) d \sigma \\
& \quad \leq \frac{3}{16 \pi R} \iint_{\sigma(\mathcal{C}, R)}\left|\frac{\partial f}{\partial \rho}\right|(x, y, z) d \sigma+\frac{1}{8 \pi R} \iint_{\sigma(\mathcal{C}, R)}\left|\frac{\partial f}{\partial \rho}\right|(x, y, z) d \sigma \\
& \quad=\frac{5}{16 \pi R} \iint_{\sigma(\mathcal{C}, R)}\left|\frac{\partial f}{\partial \rho}\right|(x, y, z) d \sigma,
\end{aligned}
$$

which implies the desired result.

Corollary 2.4 ([17]) Consider a set $I \subset \mathbb{R}^{2}$ with $D(C, R) \subset I^{\circ}$. Suppose that the mapping $f: D(C, R) \rightarrow \mathbb{R}$ has continuous partial derivatives in the disk $D(C, R)$ with respect to the variables $r$ and $\theta$ in polar coordinates. If for any constant $\theta \in[0,2 \pi]$, the function $\left|\frac{\partial f}{\partial r}\right|$ is convex with respect to the variable $r$ on $[0, R]$ then

$$
\begin{aligned}
& \left|\frac{1}{2 \pi R} \int_{\partial(C, R)} f(\gamma) d l(\gamma)-\frac{1}{\pi R^{2}} \iint_{D(C, R)} f(x, y) d x d y\right| \leq \frac{1}{6 \pi} \int_{\partial(C, R)}\left|\frac{\partial f}{\partial r}\right|(\gamma) d l(\gamma), \\
& \left|\frac{1}{\pi R^{2}} \iint_{D(C, R)} f(x, y) d x d y-f(C)\right| \leq \frac{2}{3 \pi} \int_{\partial(C, R)}\left|\frac{\partial f}{\partial r}\right|(\gamma) d l(\gamma) .
\end{aligned}
$$

Remark 2.5 In the proof of Theorem 2.3, we can find the following inequality:

$$
\begin{aligned}
& \left|\frac{1}{4 \pi R^{2}} \iint_{\sigma(\mathcal{C}, R)} f(x, y, z) d \sigma-f(\mathcal{C})\right| \\
& \quad \leq \frac{R}{2}\left|\frac{\partial f}{\partial \rho}\right|(\mathcal{C})+\frac{1}{8 \pi R} \iint_{\sigma(\mathcal{C}, R)}\left|\frac{\partial f}{\partial \rho}\right|(x, y, z) d \sigma .
\end{aligned}
$$

Although (18) is not sharp, if we consider $f(x, y, z)=\sqrt{x^{2}+y^{2}+z^{2}}$ for $x, y, z \in \overline{\mathcal{B}}(\mathcal{C}, R)$, we will find that inequality (21) is sharp. 
Remark 2.6 If we drop out the convexity condition of $\left|\frac{\partial f}{\partial \rho}\right|$ in Theorems 2.2, 2.3, and consider the condition

$$
\left\|\frac{\partial f}{\partial \rho}\right\|_{\infty_{\bar{B}(\mathcal{C}, R)}}=\sup _{w \in \bar{B}(\mathcal{C}, R)}|f(w)|<\infty,
$$

instead of that, then we get the following Ostrowski type inequalities (see $[19,20])$ on a closed ball:

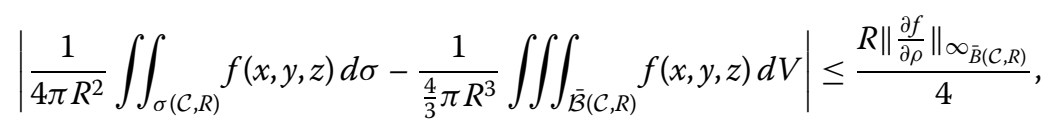

and

$$
\left|\frac{1}{\frac{4}{3} \pi R^{3}} \iiint_{\overline{\mathcal{B}}(\mathcal{C}, R)} f(x, y, z) d V-f(\mathcal{C})\right| \leq R\left\|\frac{\partial f}{\partial \rho}\right\|_{\infty_{\bar{B}(\mathcal{C}, R)}} .
$$

\section{Lipschitz functions}

In this section we consider Lipschitz functions with respect to the Euclidian norm to obtain some trapezoid and mid-point type inequalities on $\overline{\mathcal{B}}(\mathcal{C}, R)$.

Definition 3.1 ([21]) A function $f: \mathcal{V} \subset \mathbb{R}^{3} \rightarrow \mathbb{R}$ is said to satisfy a Lipschitz condition (briefly, $f$ is $\mathcal{L}$-Lipschitz) on $\mathcal{V}$ with respect to a norm $\|\cdot\|$, if there exists a constant $\mathcal{L}>0$ such that

$$
|f(x)-f(y)| \leq \mathcal{L}\|x-y\|
$$

for any $x, y \in \mathcal{V}$.

If $f: \overline{\mathcal{B}}(\mathcal{C}, R)$ is Lipschitz with respect to the Euclidian norm with the constant $\mathcal{L}>$ 0 , then for any $x=\left(a+\rho_{1} \cos \theta_{1} \sin \varphi_{1}, b+\rho_{1} \sin \theta_{1} \sin \varphi_{1}, c+\rho_{1} \cos \varphi_{1}\right)$ and $y=(a+$ $\left.\rho_{2} \cos \theta_{2} \sin \varphi_{2}, b+\rho_{2} \sin \theta_{2} \sin \varphi_{2}, c+\rho_{2} \cos \varphi_{2}\right)$, with some calculations we obtain that

$$
|f(x)-f(y)| \leq \mathcal{L} \sqrt{\rho_{1}^{2}+\rho_{2}^{2}-2 \rho_{1} \rho_{2} \mathcal{M}\left(\varphi_{1}, \varphi_{2}, \theta_{1}, \theta_{2}\right)},
$$

where $M\left(\varphi_{1}, \varphi_{2}, \theta_{1}, \theta_{2}\right)=\left[\sin \varphi_{1} \sin \varphi_{2} \cos \left(\theta_{1}-\theta_{2}\right)+\cos \varphi_{1} \cos \varphi_{2}\right], \rho_{1}, \rho_{2} \in[0, R], \theta_{1}, \theta_{2} \in$ $[0,2 \pi]$ and $\varphi_{1}, \varphi_{2} \in[0, \pi]$. Also it is obvious that if $f: \mathcal{V} \subseteq \mathbb{R}^{3} \rightarrow \mathbb{R}$ is Lipschitz with a constant $\mathcal{L}>0$ on $\mathcal{V}$, then it is continuous and so integrable on $\mathcal{V}$. We need the following result.

Lemma 3.2 For any $\varphi_{i} \in[0, \pi]$ and $\theta_{i} \in[0,2 \pi](i \in\{1,2\})$ we have

$$
-1 \leq \cos \left(\varphi_{1}+\varphi_{2}\right) \leq \mathcal{M}\left(\varphi_{1}, \varphi_{2}, \theta_{1}, \theta_{2}\right) \leq \cos \left(\varphi_{1}-\varphi_{2}\right) \leq 1 .
$$

Proof For any $\theta_{1}, \theta_{2} \in[0,2 \pi]$ it is obvious that $\cos \left(\theta_{1}-\theta_{2}\right) \leq 1$. On the other hand, since for any $\varphi_{1}, \varphi_{2} \in[0, \pi], \sin \varphi_{1} \sin \varphi_{2}$ is nonnegative,

$$
\sin \varphi_{1} \sin \varphi_{2} \cos \left(\theta_{1}-\theta_{2}\right) \leq \sin \varphi_{1} \sin \varphi_{1} .
$$


So

$$
\begin{aligned}
\mathcal{M}\left(\varphi_{1}, \varphi_{2}, \theta_{1}, \theta_{2}\right) & =\sin \varphi_{1} \sin \varphi_{2} \cos \left(\theta_{1}-\theta_{2}\right)+\cos \varphi_{1} \cos \varphi_{2} \\
& \leq \sin \varphi_{1} \sin \varphi_{2}+\cos \varphi_{1} \cos \varphi_{2}=\cos \left(\varphi_{1}-\varphi_{2}\right) \leq 1 .
\end{aligned}
$$

Similarly, we can prove that $\mathcal{M}\left(\varphi_{1}, \varphi_{2}, \theta_{1}, \theta_{2}\right) \geq \cos \left(\varphi_{1}+\varphi_{2}\right) \geq-1$.

The following trapezoid type inequality related to (1) for $\mathcal{L}$-Lipschitz functions on $\overline{\mathcal{B}}(\mathcal{C}, R)$ holds.

Theorem 3.3 Letf $: \overline{\mathcal{B}}(\mathcal{C}, R) \rightarrow \mathbb{R}$ be an $\mathcal{L}$-Lipschitz function. Then

$$
\left|\frac{1}{4 \pi R^{2}} \iint_{\sigma(\mathcal{C}, R)} f(x, y, z) d \sigma-\frac{1}{\frac{4}{3} \pi R^{3}} \iiint_{\overline{\mathcal{B}}(\mathcal{C}, R)} f(x, y, z) d V\right| \leq \frac{\mathcal{L} R}{4} .
$$

Inequality (22) is sharp.

Proof Since $f$ is Lipschitz with constant $\mathcal{L}>0$ on $\overline{\mathcal{B}}(\mathcal{C}, R)$, we get

$$
\begin{aligned}
& \mid \int_{0}^{2 \pi} \quad \int_{0}^{\pi} \int_{0}^{R} f(a+\rho \cos \theta \sin \varphi, b+\rho \sin \theta \cos \varphi, c+\rho \cos \varphi) \rho^{2} \sin \varphi d \rho d \varphi d \theta \\
& \quad-\int_{0}^{2 \pi} \int_{0}^{\pi} \int_{0}^{R} f(a+R \cos \theta \sin \varphi, b+R \sin \theta \cos \varphi, c+R \cos \varphi) \rho^{2} \sin \varphi d \rho d \varphi d \theta \mid \\
& \leq \mathcal{L} \int_{0}^{2 \pi} \int_{0}^{\pi} \int_{0}^{R}\|((\rho-R) \cos \theta \sin \varphi,(\rho-R) \sin \theta \sin \varphi,(\rho-R) \cos \varphi)\| \\
& \quad \times \rho^{2} \sin \varphi d \rho d \varphi d \theta \\
& =\mathcal{L} \int_{0}^{2 \pi} \int_{0}^{\pi} \int_{0}^{R}(R-\rho) \rho^{2} \sin \varphi d \rho d \varphi d \theta=\frac{\mathcal{L} \pi R^{4}}{3} .
\end{aligned}
$$

Now by replacing (10) and (11) in (23) and then dividing the result by $\frac{4}{3} \pi R^{3}$, we deduce the desired result.

To prove the sharpness of (22), consider the function $f: \overline{\mathcal{B}}(\mathcal{C}, R) \rightarrow \mathbb{R}$ defined by

$$
f(a+\rho \cos \theta \sin \varphi, b+\rho \sin \theta \cos \varphi, c+\rho \cos \varphi)=\mathcal{L}(R-\rho),
$$

for $\mathcal{L}>0, \rho \in[0, R], \varphi \in[0, \pi]$, and $\theta \in[0,2 \pi]$. The function $f$ is Lipschitz with constant $\mathcal{L}$. Consider $x=\left(a+\rho_{1} \cos \theta_{1} \sin \varphi_{1}, b+\rho_{1} \sin \theta_{1} \sin \varphi_{1}, c+\rho_{1} \cos \varphi_{1}\right)$ and $y=(a+$ $\left.\rho_{2} \cos \theta_{2} \sin \varphi_{2}, b+\rho_{2} \sin \theta_{2} \sin \varphi_{2}, c+\rho_{2} \cos \varphi_{2}\right)$, for $\rho_{1}, \rho_{2} \in[0, R], \varphi_{1}, \varphi_{2} \in[0, \pi], \theta_{1}, \theta_{2} \in$ $[0,2 \pi]$. Then by Lemma 3.2 we have

$$
\begin{aligned}
\mid f(x) & -f(y) \mid \\
= & \mid f\left(a+\rho_{1} \cos \theta_{1} \sin \varphi_{1}, b+\rho_{1} \sin \theta_{1} \cos \varphi_{1}, c+\rho_{1} \cos \varphi_{1}\right) \\
& -f\left(a+\rho_{2} \cos \theta_{2} \sin \varphi_{2}, b+\rho_{2} \sin \theta_{2} \cos \varphi_{2}, c+\rho_{2} \cos \varphi_{2}\right) \mid \\
= & \mathcal{L}\left|\rho_{2}-\rho_{1}\right|=\mathcal{L} \sqrt{\rho_{1}^{2}+\rho_{2}^{2}-2 \rho_{1} \rho_{2}} \leq \mathcal{L} \sqrt{\rho_{1}^{2}+\rho_{2}^{2}-2 \rho_{1} \rho_{2} \mathcal{M}\left(\varphi_{1}, \varphi_{2}, \theta_{1}, \theta_{2}\right)}
\end{aligned}
$$




$$
\begin{aligned}
= & \mathcal{L} \|\left(a+\rho_{1} \cos \theta_{1} \sin \varphi_{1}, b+\rho_{1} \sin \theta_{1} \cos \varphi_{1}, c+\rho_{1} \cos \varphi_{1}\right) \\
& -\left(a+\rho_{2} \cos \theta_{2} \sin \varphi_{2}, b+\rho_{2} \sin \theta_{2} \cos \varphi_{2}, c+\rho_{2} \cos \varphi_{2}\right)\|=\mathcal{L}\| x-y \| .
\end{aligned}
$$

It is not hard to see that $f(a+\rho \cos \theta \sin \varphi, b+\rho \sin \theta \cos \varphi, c+\rho \cos \varphi) \geq 0$ for all $0 \leq \rho \leq$ $R, 0 \leq \varphi \leq \pi$, and $0 \leq \theta \leq 2 \pi$. Also for the case $\rho=R$, we have $f(a+R \cos \theta \sin \varphi, b+$ $R \sin \theta \cos \varphi, c+R \cos \varphi)=0$. So we have

$$
\begin{gathered}
\left|\frac{1}{4 \pi R^{2}} \iint_{\sigma(\mathcal{C}, R)} f(x, y, z) d \sigma-\frac{1}{\frac{4}{3} \pi R^{3}} \iiint_{\overline{\mathcal{B}}(\mathcal{C}, R)} f(x, y, z) d V\right| \\
=\frac{1}{\frac{4}{3} \pi R^{3}} \iiint_{\overline{\mathcal{B}}(\mathcal{C}, R)} f(x, y, z) d V \\
=\frac{\mathcal{L}}{\frac{4}{3} \pi R^{3}} \int_{0}^{2 \pi} \int_{0}^{\pi} \int_{0}^{R}(R-\rho) \rho^{2} \sin \varphi d \rho d \varphi d \theta=\frac{\mathcal{L} R}{4} .
\end{gathered}
$$

For $\mathcal{L}$-Lipschitz functions we can obtain a mid-point type inequality as follows:

Theorem 3.4 Letf $: \overline{\mathcal{B}}(\mathcal{C}, R) \rightarrow \mathbb{R}$ be an $\mathcal{L}$-Lipschitz function. Then

$$
\left|\frac{1}{\frac{4}{3} \pi R^{3}} \iiint_{\overline{\mathcal{B}}(\mathcal{C}, R)} f(x, y, z) d V-f(\mathcal{C})\right| \leq \frac{3 \mathcal{L} R}{4} .
$$

Inequality (24) is sharp.

Proof Since the function $f$ is $\mathcal{L}$-Lipschitz on $\overline{\mathcal{B}}(\mathcal{C}, R)$, we have

$$
\begin{aligned}
& |f(a+\rho \cos \theta \sin \varphi, b+\rho \sin \theta \cos \varphi, c+\rho \cos \varphi)-f(\mathcal{C})| \\
& \quad \leq \mathcal{L}\|(\rho \cos \theta \sin \varphi, \rho \sin \theta \cos \varphi, \rho \cos \varphi)\|=\mathcal{L} \rho,
\end{aligned}
$$

for all $\rho \in[0, R], \varphi \in[0, \pi]$, and $\theta \in[0,2 \pi]$. It follows that

$$
\begin{aligned}
& \mid \int_{0}^{2 \pi} \quad \int_{0}^{\pi} \int_{0}^{R} f(a+\rho \cos \theta \sin \varphi, b+\rho \sin \theta \cos \varphi, c+\rho \cos \varphi) \rho^{2} \sin \varphi d \rho d \varphi d \theta \\
& \quad-\int_{0}^{2 \pi} \int_{0}^{\pi} \int_{0}^{R} f(a, b, c) \rho^{2} \sin \varphi d \rho d \varphi d \theta \mid \\
& \leq \int_{0}^{2 \pi} \int_{0}^{\pi} \int_{0}^{R}|f(a+\rho \cos \theta \sin \varphi, b+\rho \sin \theta \cos \varphi, c+\rho \cos \varphi)-f(\mathcal{C})| \\
& \quad \times \rho^{2} \sin \varphi d \rho d \varphi d \theta \\
& \leq \mathcal{L} \int_{0}^{2 \pi} \int_{0}^{\pi} \int_{0}^{R} \rho^{3} \sin \varphi d \rho d \varphi d \theta=\mathcal{L} \pi R^{4} .
\end{aligned}
$$

So we obtain that

$$
\left|\iiint_{\overline{\mathcal{B}}(\mathcal{C}, R)} f(x, y, z) d V-\frac{4}{3} \pi R^{3} f(\mathcal{C})\right| \leq \mathcal{L} \pi R^{4},
$$

which implies the desired result. 
Now consider the function $f: \overline{\mathcal{B}}(\mathcal{C}, R) \rightarrow \mathbb{R}$ defined by

$$
f(a+\rho \cos \theta \sin \varphi, b+\rho \sin \theta \cos \varphi, c+\rho \cos \varphi)=\mathcal{L} \rho,
$$

for $\mathcal{L}>0,0 \leq \rho \leq R, 0 \leq \varphi \leq \pi$, and $0 \leq \theta \leq 2 \pi$. It is obvious that $f(\mathcal{C})=0$. By a similar method used in the proof of Theorem 3.3, the function $f$ is $\mathcal{L}$-Lipschitz. So we have

$$
\left|\frac{1}{\frac{4}{3} \pi R^{3}} \iiint_{\overline{\mathcal{B}}(\mathcal{C}, R)} f(x, y, z) d V-f(\mathcal{C})\right|=\frac{\mathcal{L}}{\frac{4}{3} \pi R^{3}} \int_{0}^{2 \pi} \int_{0}^{\pi} \int_{0}^{R} \rho^{3} \sin \varphi d \rho d \varphi d \theta=\frac{3 \mathcal{L} R}{4},
$$

showing that inequality (24) is sharp.

Remark 3.5 Consider an open set $\mathcal{V} \subset \mathbb{R}^{3}$ including $\overline{\mathcal{B}}(\mathcal{C}, R)$. For convex function $f$ defined on $\mathcal{V}$, from Theorem D of Sect. 41 in [21] we have that $f$ is $\mathcal{L}$-Lipschitz on $\overline{\mathcal{B}}(\mathcal{C}, R)$ and so from inequalities (22) and (24), along with inequality (1), we get the following results:

$$
0 \leq \frac{1}{4 \pi R^{2}} \iint_{\sigma(\mathcal{C}, R)} f(x, y, z) d \sigma-\frac{1}{\frac{4}{3} \pi R^{3}} \iiint_{\overline{\mathcal{B}}(\mathcal{C}, R)} f(x, y, z) d V \leq \frac{\mathcal{L} R}{3},
$$

and

$$
0 \leq \frac{1}{\frac{4}{3} \pi R^{3}} \iiint_{\overline{\mathcal{B}}(\mathcal{C}, R)} f(x, y, z) d V-f(\mathcal{C}) \leq \frac{2 \mathcal{L} R}{3} .
$$

In the following, as an example we obtain a Lipschitz constant $\mathcal{L}$ for a real-valued function defined on a closed ball in $\mathbb{R}^{3}$.

Example 3.6 Consider $W=f(x, y, z)=(x-a)^{n}+(y-b)^{n}+(z-c)^{n}, n \in \mathbb{N},(x, y, z) \in \overline{\mathcal{B}}(\mathcal{C}, R)$. To find a Lipschitz constant for $f$, we will do some calculations as follows.For $A, B \in$ $\overline{\mathcal{B}}(\mathcal{C}, R)$, consider the path $\psi:[0,1] \rightarrow \overline{\mathcal{B}}(\mathcal{C}, R)$ from $B$ to $A$ in $\overline{\mathcal{B}}(\mathcal{C}, R)$ as

$$
\psi(t)=t A+(1-t) B
$$

for $t \in[0,1]$. Now using the fundamental theorem of calculus, we obtain that

$$
|f(A)-f(B)|=|f(\psi(1))-f(\psi(0))|=\left|\int_{0}^{1} \frac{d f(\psi(t))}{d t} d t\right|
$$

On the other hand, from the chain rule for differentiation, we get

$$
\frac{d f(\psi(t))}{d t}=\nabla f(\psi(t)) \cdot \frac{d \psi}{d t}=\nabla f(\psi(t))(A-B)
$$

where $\nabla f$ is the gradient vector of $f$. So using the Euclidean norm $\|\cdot\|$, we obtain

$$
\begin{aligned}
\left|\int_{0}^{1} \frac{d f(\psi(t))}{d t} d t\right| & =\left|\int_{0}^{1} \nabla f(\psi(t))(A-B) d t\right| \leq\|A-B\| \int_{0}^{1}\|\nabla f(\psi(t))\| d t \\
& \leq\|A-B\| \sup _{u \in \overline{\mathcal{B}}(\mathcal{C}, R)}\|\nabla f(u)\|,
\end{aligned}
$$


which implies

$$
|f(A)-f(B)| \leq\|A-B\| \sup _{u \in \overline{\mathcal{B}}(\mathcal{C}, R)}\|\nabla f(u)\| .
$$

This shows that $\mathcal{L}=\sup _{u \in \overline{\mathcal{B}}(\mathcal{C}, R)}\|\nabla f(u)\|$ (if it exists) is a Lipschitz constant for $f$. Now for any $w=(x, y, z) \in \overline{\mathcal{B}}(\mathcal{C}, R)$, we have

$$
\nabla f(w)=n\left((x-a)^{n-1},(y-b)^{n-1},(z-c)^{n-1}\right),
$$

and then

$$
\begin{aligned}
\|\nabla f(u)\| & =n \sqrt{\left((x-a)^{n-1}\right)^{2}+\left((y-b)^{n-1}\right)^{2}+\left((z-c)^{n-1}\right)^{2}} \\
& \leq n \sqrt{\left((x-a)^{2}+(y-b)^{2}+(z-c)^{2}\right)^{n-1}}=n R^{n-1} .
\end{aligned}
$$

So we can choose $\mathcal{L}=\sup _{u \in \overline{\mathcal{B}}(\mathcal{C}, R)}\|\nabla f(u)\|=n R^{n-1}$ as a Lipschitz constant for $f$ on $\overline{\mathcal{B}}(\mathcal{C}, R)$.

Using the above example, we have the following result:

Example 3.7 For $n \in \mathbb{N} \backslash\{1\}$, consider the function $f(\rho, \varphi, \theta)=\left(x_{0}-\rho\right)^{n}+\left(y_{0}-\rho\right)^{n}+\left(z_{0}-\rho\right)^{n}$ defined on $\overline{\mathcal{B}}\left(\left(x_{0}, y_{0}, z_{0}\right), R\right)$ such that $x_{0}, y_{0}, z_{0}>0,0<R \leq \min \left\{x_{0}, y_{0}, z_{0}\right\}$ and $0 \leq \rho \leq R$. It follows that

$$
\nabla\left(\frac{\partial f}{\partial \rho}\right)(\rho, \varphi, \theta)=n(n-1)\left(\left(x_{0}-\rho\right)^{n-2}+\left(y_{0}-\rho\right)^{n-2}+\left(z_{0}-\rho\right)^{n-2}, 0,0\right),
$$

and then

$$
\mathcal{L}=n(n-1)\left(x_{0}^{n-2}+y_{0}^{n-2}+z_{0}^{n-2}\right)
$$

is a Lipschitz constant for $\nabla\left(\frac{\partial f}{\partial \rho}\right)$. On the other hand, it is not hard to prove that

$$
\left|\frac{1}{4 \pi R^{2}} \iint_{\sigma(\mathcal{C}, R)} f(x, y, z) d \sigma-f(\mathcal{C})\right| \leq \frac{\mathcal{L} R^{2}}{2} .
$$

So by (25), we have the following numerical inequality:

$$
\begin{aligned}
& \left|\left(\left(x_{0}-R\right)^{n}+\left(y_{0}-R\right)^{n}+\left(z_{0}-R\right)^{n}\right)-\left(x_{0}^{n}+y_{0}^{n}+z_{0}^{n}\right)\right| \\
& \quad \leq \frac{n(n-1)\left(x_{0}^{n-2}+y_{0}^{n-2}+z_{0}^{n-2}\right) R^{2}}{2} .
\end{aligned}
$$

Remark 3.8 For any function $f: \overline{\mathcal{B}}(\mathcal{C}, R) \rightarrow \mathbb{R}$, we can apply the structure mentioned in the above example to obtain a Lipschitz constant $\mathcal{L}=\sup _{z \in \overline{\mathcal{B}}(\mathcal{C}, R)}\|\nabla f(z)\|$ with respect to the Euclidian norm $\|\cdot\|$, provided that the gradient vector of $f$ exists everywhere in $\overline{\mathcal{B}}(\mathcal{C}, R)$ and also $\mathcal{L}<\infty$.

Remark 3.9 In Theorems 3.3 and 3.4, if we consider that $\frac{\partial f}{\partial \rho}: \overline{\mathcal{B}}(\mathcal{C}, R) \rightarrow \mathbb{R}$ is $\mathcal{L}$-Lipschitz and $f: \overline{\mathcal{B}}(\mathcal{C}, R) \rightarrow \mathbb{R}$ is integrable, then by (13) and (19) we can obtain (the details are 
omitted)

$$
\left|\frac{1}{4 \pi R^{2}} \iint_{\sigma(\mathcal{C}, R)} f(x, y, z) d \sigma-\frac{1}{\frac{4}{3} \pi R^{3}} \iiint_{\overline{\mathcal{B}}(\mathcal{C}, R)} f(x, y, z) d V\right| \leq \frac{1}{5} \mathcal{L} R^{2}
$$

and

$$
\left|\frac{1}{\frac{4}{3} \pi R^{3}} \iiint_{\overline{\mathcal{B}}(\mathcal{C}, R)} f(x, y, z) d V-f(\mathcal{C})\right| \leq \frac{7}{10} \mathcal{L} R^{2}
$$

\section{Bounded functions}

In the last section we investigate trapezoid and mid-point type inequalities where considered functions are bounded.

Theorem 4.1 Suppose that $\mathcal{V} \subset \mathbb{R}^{3}, \overline{\mathcal{B}}(\mathcal{C}, R) \subset \mathcal{V}^{\circ}$ and $f: \overline{\mathcal{B}}(\mathcal{C}, R) \rightarrow \mathbb{R}$ has continuous partial derivatives with respect to the variables $\rho, \varphi$, and $\theta$ on $\overline{\mathcal{B}}(\mathcal{C}, R)$ in spherical coordinates. If $\frac{\partial f}{\partial \rho}$ is bounded on $\overline{\mathcal{B}}(\mathcal{C}, R)$, then

$$
\begin{aligned}
& \left|\frac{1}{4 \pi R^{2}} \iint_{\sigma(\mathcal{C}, R)} f(x, y, z) d \sigma-\frac{1}{\frac{4}{3} \pi R^{3}} \iiint_{\overline{\mathcal{B}}(\mathcal{C}, R)} f(x, y, z) d V\right| \\
& \quad \leq\left(\frac{\mathcal{U}_{B}-\mathcal{L}_{B}+\left|\mathcal{L}_{B}+\mathcal{U}_{B}\right|}{8}\right) R
\end{aligned}
$$

where $\mathcal{L}_{B}$ and $\mathcal{U}_{B}$ are lower and upper bounds of $\frac{\partial f}{\partial \rho}$ on $\overline{\mathcal{B}}(\mathcal{C}, R)$, respectively.

Proof Consider $\mathcal{U}_{B}$ and $\mathcal{L}_{B}$ as the upper and lower bounds of an arbitrary function $g$ defined on a set $\mathcal{V} \subset \mathbb{R}^{3}$, respectively. Then for all $x, y, z \in \mathcal{V}$, we have

$$
\mathcal{L}_{B}-\frac{\mathcal{L}_{B}+\mathcal{U}_{B}}{2} \leq g(x, y, z)-\frac{\mathcal{L}_{B}+\mathcal{U}_{B}}{2} \leq \mathcal{U}_{B}-\frac{\mathcal{L}_{B}+\mathcal{U}_{B}}{2},
$$

which implies that

$$
\left|g(x, y, z)-\frac{\mathcal{L}_{B}+\mathcal{U}_{B}}{2}\right| \leq \frac{\mathcal{U}_{B}-\mathcal{L}_{B}}{2},
$$

for all $x, y, z \in \mathcal{V}$. On the other hand, from (13) we get

$$
\begin{aligned}
\int_{0}^{2 \pi} & \int_{0}^{\pi} \int_{0}^{R} \frac{\partial f}{\partial \rho}(a+\rho \cos \theta \sin \varphi, b+\rho \sin \theta \sin \varphi, c+\rho \cos \varphi) \rho^{3} \sin \varphi d \rho d \varphi d \theta \\
& -\int_{0}^{2 \pi} \int_{0}^{\pi} \int_{0}^{R} \frac{\mathcal{L}_{B}+\mathcal{U}_{B}}{2} \rho^{3} \sin \varphi d \rho d \varphi d \theta \\
= & \int_{0}^{2 \pi} \int_{0}^{\pi} R^{3} f(a+R \cos \theta \sin \varphi, b+R \sin \theta \sin \varphi, c+R \cos \varphi) d \varphi d \theta \\
& -3 \int_{0}^{2 \pi} \int_{0}^{\pi} \int_{0}^{R} f(a+\rho \cos \theta \sin \varphi, b+\rho \sin \theta \sin \varphi, c+\rho \cos \varphi) \rho^{2} \sin \varphi d \rho d \varphi d \theta \\
& -\pi R^{4} \frac{\mathcal{L}_{B}+\mathcal{U}_{B}}{2} .
\end{aligned}
$$


Now if in (27) we consider $g=\frac{\partial f}{\partial \rho}, \mathcal{V}=\overline{\mathcal{B}}(\mathcal{C}, R)$, and utilize Lemma 2.1, then we obtain that

$$
\begin{aligned}
\left|R \iint_{\sigma(\mathcal{C}, R)} f(x, y, z) d \sigma-3 \iiint_{\overline{\mathcal{B}}(\mathcal{C}, R)} f(x, y, z) d V-\pi R^{4} \frac{\mathcal{L}_{B}+\mathcal{U}_{B}}{2}\right| \\
\leq \int_{0}^{2 \pi} \int_{0}^{\pi} \int_{0}^{R}\left|\frac{\partial f}{\partial \rho}(a+\rho \cos \theta \sin \varphi, b+\rho \sin \theta \sin \varphi, c+\rho \cos \varphi)-\frac{\mathcal{L}_{B}+\mathcal{U}_{B}}{2}\right| \\
\quad \times \rho^{3} \sin \varphi d \rho d \varphi d \theta \\
\leq \int_{0}^{2 \pi} \int_{0}^{\pi} \int_{0}^{R}\left|\frac{\mathcal{U}_{B}-\mathcal{L}_{B}}{2}\right| \rho^{3} \sin \varphi d \rho d \varphi d \theta=\frac{\mathcal{U}_{B}-\mathcal{L}_{B}}{2} \pi R^{4} .
\end{aligned}
$$

Finally, by the use of the triangle inequality and dividing the result by $4 \pi R^{3}$, we obtain inequality (26).

Theorem 4.2 Suppose that $\mathcal{V} \subset \mathbb{R}^{3}, \overline{\mathcal{B}}(\mathcal{C}, R) \subset \mathcal{V}^{\circ}$ and $f: \overline{\mathcal{B}}(\mathcal{C}, R) \rightarrow \mathbb{R}$ has continuous partial derivatives with respect to the variables $\rho, \varphi$, and $\theta$ on $\overline{\mathcal{B}}(\mathcal{C}, R)$ in spherical coordinates. If $\frac{\partial f}{\partial \rho}$ is bounded on $\overline{\mathcal{B}}(\mathcal{C}, R)$, then

$$
\left|\frac{1}{4 \pi R^{2}} \iint_{\sigma(\mathcal{C}, R)} f(x, y, z) d \sigma-f(\mathcal{C})\right| \leq\left(\frac{\mathcal{U}_{B}-\mathcal{L}_{B}+\left|\mathcal{L}_{B}+\mathcal{U}_{B}\right|}{2}\right) R
$$

where $\mathcal{L}_{B}$ and $\mathcal{U}_{B}$ are lower and upper bounds of $\frac{\partial f}{\partial \rho}$ on $\overline{\mathcal{B}}(\mathcal{C}, R)$, respectively.

Proof Consider $\mathcal{L}_{B}$ and $\mathcal{U}_{B}$ as the upper and lower bounds of $\frac{\partial f}{\partial \rho}$. By (19), the following relations hold:

$$
\begin{aligned}
\int_{0}^{2 \pi} & \int_{0}^{\pi} \int_{0}^{R}\left[\frac{\partial f}{\partial \rho}(a+\rho \cos \theta \sin \varphi, b+\rho \sin \theta \cos \varphi, c+\rho \cos \varphi)-\frac{\mathcal{L}_{B}+\mathcal{U}_{B}}{2}\right] \\
& \times \sin \varphi d \rho d \varphi d \theta \\
= & \int_{0}^{2 \pi} \int_{0}^{\pi} f(a+R \cos \theta \sin \varphi, b+R \sin \theta \sin \varphi, c+R \cos \varphi) \sin \varphi d \varphi d \theta \\
& -\int_{0}^{2 \pi} \int_{0}^{\pi} f(\mathcal{C}) \sin \varphi d \varphi d \theta-\frac{\mathcal{L}_{B}+\mathcal{U}_{B}}{2} R \int_{0}^{2 \pi} \int_{0}^{\pi} \sin \varphi d \varphi d \theta \\
= & \frac{1}{R^{2}} \iint_{\sigma(\mathcal{C}, R)} f(x, y, z) d \sigma-4 \pi f(\mathcal{C})-2 \pi\left(\mathcal{L}_{B}+\mathcal{U}_{B}\right) R .
\end{aligned}
$$

This implies that

$$
\begin{aligned}
& \left|\frac{1}{4 \pi R^{2}} \iint_{\sigma(\mathcal{C}, R)} f(x, y, z) d \sigma-f(\mathcal{C})-\frac{\mathcal{L}_{B}+\mathcal{U}_{B}}{2} R\right| \\
& \leq \frac{1}{4 \pi} \int_{0}^{2 \pi} \int_{0}^{\pi} \int_{0}^{R}\left|\frac{\partial f}{\partial \rho}(a+\rho \cos \theta \sin \varphi, b+\rho \sin \theta \sin \varphi, c+\rho \cos \varphi)-\frac{\mathcal{L}_{B}+\mathcal{U}_{B}}{2}\right| \\
& \quad \times \sin \varphi d \rho d \varphi d \theta=\frac{\mathcal{U}_{B}-\mathcal{L}_{B}}{2} R .
\end{aligned}
$$


Finally, by using the triangle inequality, we get

$$
\left|\frac{1}{4 \pi R^{2}} \iint_{\sigma(\mathcal{C}, R)} f(x, y, z) d \sigma-f(\mathcal{C})\right| \leq\left(\frac{\mathcal{U}_{B}-\mathcal{L}_{B}+\left|\mathcal{L}_{B}+\mathcal{U}_{B}\right|}{2}\right) R .
$$

Remark 4.3 If $f: \overline{\mathcal{B}}(\mathcal{C}, R) \rightarrow \mathbb{R}$ is a convex function and bounded from above on $\overline{\mathcal{B}}(\mathcal{C}, R)$ $\left(\mathcal{U}_{B}\right.$ exists), then $f$ is bounded on $\overline{\mathcal{B}}(\mathcal{C}, R)$ because for an arbitrary $X \in \overline{\mathcal{B}}(0, R)$ and $\mathcal{C}=$ $\frac{1}{2}(X+\mathcal{C})+\frac{1}{2}(-X+\mathcal{C})$, from the convexity of $f$ we have $2 f(\mathcal{C})-f(-X+\mathcal{C}) \leq f(X+\mathcal{C})$. This implies that $2 f(\mathcal{C})-\mathcal{U}_{B} \leq f(X+\mathcal{C})$ where $X+\mathcal{C}$ and $-X+\mathcal{C}$ belong to $\overline{\mathcal{B}}(\mathcal{C}, R)$. Now it is enough to set $\mathcal{L}_{B}=2 f(\mathcal{C})-\mathcal{U}_{B}$.

So if $\frac{\partial f}{\partial \rho}: \overline{\mathcal{B}}(\mathcal{C}, R) \rightarrow \mathbb{R}$ is convex and bounded from above, then by (26), (28), and (1), the following inequalities hold:

$$
\begin{aligned}
0 & \leq \frac{1}{4 \pi R^{2}} \iint_{\sigma(\mathcal{C}, R)} f(x, y, z) d \sigma-\frac{1}{\frac{4}{3} \pi R^{3}} \iiint_{\overline{\mathcal{B}}(\mathcal{C}, R)} f(x, y, z) d V \\
& \leq\left(\frac{\mathcal{U}_{B}-\mathcal{L}_{B}+\left|\mathcal{L}_{B}+\mathcal{U}_{B}\right|}{8}\right) R,
\end{aligned}
$$

and

$$
0 \leq \frac{1}{\frac{4}{3} \pi R^{3}} \iiint_{\overline{\mathcal{B}}(\mathcal{C}, R)} f(x, y, z) d V-f(\mathcal{C}) \leq\left(\frac{\mathcal{U}_{B}-\mathcal{L}_{B}+\left|\mathcal{L}_{B}+\mathcal{U}_{B}\right|}{2}\right) R
$$

where $\mathcal{L}_{B}$ and $\mathcal{U}_{B}$ are lower and upper bounds of $\frac{\partial f}{\partial \rho}$ on $\overline{\mathcal{B}}(\mathcal{C}, R)$, respectively.

\section{Acknowledgements}

The author is grateful to the referee(s) and the editor for their valuable comments and suggestions.

\section{Funding}

Not applicable.

Availability of data and materials

Not applicable.

\section{Competing interests}

The author declares that he has no competing interests.

\section{Authors' contributions}

The author read and approved the final manuscript.

\section{Publisher's Note}

Springer Nature remains neutral with regard to jurisdictional claims in published maps and institutional affiliations.

Received: 7 November 2019 Accepted: 14 April 2020 Published online: 28 April 2020

\section{References}

1. Dragomir, S.S.: On Hadamard's inequality for the convex mappings defined on a ball in the space and applications. Math. Inequal. Appl. 3(2), 177-187 (2000)

2. Kirmaci, U.S.: Inequalities for differentiable mappings and applications to special means of real numbers and to midpoint formula. Appl. Math. Comput. 147(1), 137-146 (2004)

3. Dragomir, S.S., Agarwal, R.P.: Two inequalities for differentiable mappings and applications to special means of real numbers and to trapezoidal formula. Appl. Math. Lett. 11, 91-95 (1998)

4. Guessab, A., Schmeisser, G.: Convexity results and sharp error estimates in approximate multivariate integration. Math. Comput. 73, 1365-1384 (2004)

5. Guessab, A., Schmeisser, G.: Sharp integral inequalities of the Hermite-Hadamard type. J. Approx. Theory 115 260-288 (2002)

6. Bessenyei, M.: The Hermite-Hadamard inequality on simplices. Am. Math. Mon. 115(4), 339-345 (2008)

7. Chen, Y.: Multi-dimensional Hadamard's inequalities. Tamkang J. Math. 43(1), 1-10 (2012) 
8. Dragomir, S.S.: On Hadamard's inequality on a disk. J. Inequal. Pure Appl. Math. 1(1), Article ID 2 (2000)

9. Hwang, D.-Y.: Some inequalities for differentiable convex mapping with application to weighted trapezoidal formula and higher moments of random variables. Appl. Math. Comput. 217, 9598-9605 (2011)

10. Matłoka, M.: On Hadamard's inequality for $h$-convex function on a disk. Appl. Math. Comput. 235, 118-123 (2014)

11. Neuman, E.: Inequalities involving multivariate convex functions II. Proc. Am. Math. Soc. 109(4), 965-974 (1990)

12. Niculescu, C.P., Persson, L.E.: Convex Functions and Their Applications: A Contemporary Approach. CMS Books in Mathematics. Springer, Berlin (2006)

13. Nowicka, M., Witkowski, A.: Applications of the Hermite-Hadamard inequality. Math. Inequal. Appl. 19(4), 1319-1334 (2016)

14. Pečarić, J., Proschan, F., Tong, Y.L.: Convex Functions, Partial Orderings, and Statistical Applications. Academic Press, San Diego (1992)

15. Rostamian Delavar, M., Dragomir, S.S.: Weighted trapezoidal inequalities related to the area balance of a function with applications. Appl. Math. Comput. 340, 5-14 (2019)

16. Rostamian Delavar, M., Dragomir, S.S.: On $\eta$-convexity. Math. Inequal. Appl. 20, 203-216 (2017)

17. Rostamian Delavar, M., Dragomir, S.S., De La Sen, M.: Hermite-Hadamard's trapezoid and mid-point type inequalities on a disk. J. Inequal. Appl. 2019, 105 (2019)

18. Varošanec, S.: On h-convexity. J. Math. Anal. Appl. 326, 303-311 (2007)

19. Dragomir, S.S., Rassias, Th.M.: Ostrowski Type Inequalities and Applications in Numerical Integration. Kluwer Academic, Dordrecht (2002)

20. Ostrowski, A.M.: Über die Absolutabweichung einer differentiebaren Funktion von ihrem Integralmitelwert. Comment. Math. Helv. 10, 226-227 (1938)

21. Robert, A.W., Varbeg, D.E.: Convex Functions. Academic Press, New York (1973)

\section{Submit your manuscript to a SpringerOpen ${ }^{\odot}$ journal and benefit from:}

- Convenient online submission

- Rigorous peer review

- Open access: articles freely available online

- High visibility within the field

- Retaining the copyright to your article

Submit your next manuscript at $\gg$ springeropen.com 